\title{
Group decision aiding by Interval AHP with compromise and refinement
}

\author{
Masahiro Inuiguchi ${ }^{1}$ Tomoe Entani $^{2}$ \\ ${ }^{1}$ Graduate School of Engineering Science, Osaka University, Toyonaka, Osaka 560-8531, Japan \\ E-mail: inuiguti@sys.es.osaka-u.ac.jp \\ ${ }^{2}$ Graduate School of Applied Informatics, University of Hyogo, Kobe, Hyogo 650-0047, Japan \\ E-mail: entani@ai.u-hyogo.ac.jp
}

\begin{abstract}
Interval AHP was proposed to express the decision maker's vague evaluations on criteria by interval weights from a given pairwise comparison matrix. It has been extended to group decision problems. Three complementary approaches have been proposed: the perfect incorporation approach for counting out indubitably inferior alternatives and the common ground and partial incorporation approaches for finding agreeable preference between alternatives. In this paper, we enhance those approaches by working out conceivable compromise and refinement. Compromise solution and refinement solution can be found by solving linear programming problems.
\end{abstract}

Keywords: analytic hierarchy process, group decision-making, interval analysis, linear programming, dominance relation

\section{Introduction}

Analytic Hierarchy Process (AHP) [1] provides a useful tool to extract the decision maker's evaluation about the importance of criteria and alternatives. It considers the hierarchy of the evaluation but we focus on the estimation of weights of criteria at the top level of the hierarchy in this paper. Given a pairwise comparison matrix showing decision maker's judgment on the relative importance between criteria, weights of criteria are estimated. However, the human judgment is vague in nature so that the given pairwise comparison matrix is often inconsistent. The consistency index is defined and used to evaluate the reliability of the estimated weights. If the value of the consistency index is in a certain range, the estimated weights are accepted and used for the further decision analysis.

To cope with the vagueness, fuzzy and interval approaches are proposed. In many of fuzzy approaches, a pairwise comparison matrix with fuzzy components is used to represent the vagueness of human judgment on the relative importance between criteria. Then a fuzzy weight vector is estimated so as to approximate the fuzzy pairwise comparison matrix $[2,3]$. Similarly, a pairwise comparison matrix with interval components is also used to represent the vagueness of human judgment. However, a crisp weight vector consistent or nearly consistent to a given interval pairwise comparison matrix is estimated (see [4]).

On the other hand, in Interval AHP [5], an interval weight vector is estimated from a given crisp pairwise comparison matrix. This approach is based on the idea that the decision maker's evaluation is vague so that the weights of criteria are expressed by intervals and that the pairwise comparison matrix is obtained by judgments with arbitrarily selected values from the intervals. An interval weight vector covering the given pairwise comparison matrix is estimated so as to minimize its total spreads. Later Interval AHP is extended to treat interval pairwise comparison matrices [6] but we treat only a crisp pairwise comparison matrix in this paper.

In the previous paper [7], we proposed to apply Interval AHP to group decision aiding. Three approaches have been proposed: the perfect incorporation approach for counting out indubitably inferior alternatives and the common ground and partial incorporation approaches for finding agreeable preference between alternatives. However, neither compromise of each decision maker nor a refinement of the consensus dominance relation has been yet introduced into those models.

In this paper, we introduce compromises of decision makers and a refinement of the consensus dominance relation. In the perfect incorporation approach, decision makers compromise the perfect incorporation of their opinions. In the common ground approach, we refine the consensus dominance relation by considering possible change of individual opinions. In the partial incorporation approach, decision makers compromise the precision of individual opinions. By those proposed approaches, we obtain dominance relations among alternatives more useful in narrowing down the candidates. We give a few numerical examples to demonstrate the usefulness of the proposed approaches.

Differently from the soft consensus models [8] in a fuzzy environment, the group decision aiding with Interval AHP does not provide a method to achieve a consensus dominance relation or a consensus solution but finds only an agreeable part of dominance 
relation between alternatives utilizing the elasticity of individual evaluations of criteria importance. Therefore, this group decision aiding does not request the decision makers to update their pairwise comparisons. Following this policy, compromises of decision makers and a refinement of the consensus dominance relation are introduced without requesting the decision makers to update their preferences.

This paper is organized as follows. In next section, Interval AHP for crisp pairwise comparison matrix and Group Interval AHP are briefly reviewed. In Section 3, we extend the perfect incorporation, common ground and partial incorporation approaches by introducing compromise and refinement. A few numerical examples are given in Section 4. In Section 5, we describe concluding remarks.

\section{Interval AHP and Group Interval AHP}

\subsection{Interval AHP}

We introduce Interval AHP for multiple criteria decision problem with a single decision maker as the basis for treating a group decision problem with $m$ decision makers and $n$ criteria. For simplicity and the consistency of notation with subsequent sections, we define $M=\{1,2, \ldots, m\}, N=$ $\{1,2, \ldots, n\}$ and $N \backslash j=N \backslash\{j\}=\{1,2, \ldots, j-$ $1, j+1, \ldots, n\}$ for $j \in N$. In this subsection, we describe Interval AHP for the problem of decision maker $k \in M$.

In AHP, the decision problem is structured hierarchically as criteria and alternatives. At each node except leaf nodes of the hierarchical tree, a weight vector for criteria or for alternatives is obtained from a pairwise comparison matrix $A_{k}$ given by decision maker $k \in M$. We concentrate on the estimation of a weight vector for criteria with respect to the decision maker $k$.

We first describe the estimation of a weight vector $\boldsymbol{w}_{k}=\left(w_{k 1}, w_{k 2}, \ldots, w_{k n}\right)^{\mathrm{T}}$ from a given pairwise comparison matrix,

$$
A_{k}=\left[\begin{array}{ccc}
1 & \cdots & a_{k 1 n} \\
\vdots & a_{k i j} & \vdots \\
a_{k n 1} & \cdots & 1
\end{array}\right]
$$

where we assume the reciprocity, i.e., $a_{k i j}=1 / a_{k i j}$, $i, j \in N$. Because the $(i, j)$ component $a_{k i j}$ of $A_{k}$ shows the relative importance of the $i$-th criterion over the $j$-th criterion, theoretically, we have $a_{k i j}=w_{k i} / w_{k j}, i, j \in N$. If $a_{k i j}, i, j \in N$ are obtained exactly, the strong transitivity $a_{k i j}=$ $a_{k i l} a_{k l j}, i, j, l \in N$ should be satisfied. However, human evaluation is not very accurate so that the strong transitivity is not satisfied. In the conventional approach [1], $a_{k i j}, i, j \in N$ are assumed to be approximations of $w_{k i} / w_{k j} . \quad \boldsymbol{w}_{k}$ is estimated as the normalized eigenvector corresponding to the maximal eigenvalue, because the nonnegativity of the eigenvector corresponding to the maximal eigenvalue is guaranteed by Perron-Frobenius theorem. There is the other popular way to estimate $\boldsymbol{w}_{k}$. It is the geometric mean method [3]. To evaluate the consistency of the given pairwise comparison matrix, the following consistency index is used:

$$
\text { C.I. }=\frac{\lambda_{\max }-n}{n-1},
$$

where $\lambda_{\max }$ is the maximal eigenvalue of $A_{k}$. If C.I. is not greater than 0.1 , it is often considered that the obtained vector $\boldsymbol{w}_{k}$ is acceptable.

To this estimation problem, the idea of interval regression analysis [9] was applied. In this approach, we assume that decision maker $k$ 's evaluation is not very accurate to be expressed by a unique weight vector $\boldsymbol{w}_{k}$ but intrinsically vague so that the weight vector has a range. Therefore, the non-fulfillment of the strong transitivity is not regarded as inconsistency but due to the intrinsic variety of possible weight vectors. Accordingly, we consider an interval weight vector $\boldsymbol{W}_{k}=$ $\left(W_{k 1}, W_{k 2}, \ldots, W_{k n}\right)^{\mathrm{T}}$ instead of a weight vector $\boldsymbol{w}$, where $W_{k i}=\left[w_{k i}^{\mathrm{L}}, w_{k i}^{\mathrm{R}}\right], i \in N$ and $w_{k i}^{\mathrm{L}} \leq w_{k i}^{\mathrm{R}}$, $i \in N$. To fit the given pairwise comparison matrix, we require the interval weight vector $\boldsymbol{W}$ to satisfy

$$
\frac{w_{k i}^{\mathrm{L}}}{w_{k j}^{\mathrm{R}}} \leq a_{k i j} \leq \frac{w_{k i}^{\mathrm{R}}}{w_{k j}^{\mathrm{L}}}, i, j \in N, i<j .
$$

We note that by the reciprocity, $a_{k i j}=1 / a_{k i j}$, $i, j \in N$, we only consider $i, j \in N$ such that $i<j$. The set of interval weight vectors $\boldsymbol{W}_{k}$ satisfying (3) is denoted by $\mathcal{W}\left(A_{k}\right)$. Moreover, corresponding to normalization condition of $\boldsymbol{w}_{k}$ in the conventional AHP, we require the interval weight vector $\boldsymbol{W}_{k}$ to satisfy

$$
\begin{aligned}
& \sum_{j \in N \backslash i} w_{k j}^{\mathrm{R}}+w_{k i}^{\mathrm{L}} \geq 1, i \in N, \\
& \sum_{j \in N \backslash i} w_{k j}^{\mathrm{L}}+w_{k i}^{\mathrm{R}} \leq 1, \quad i \in N .
\end{aligned}
$$

(4) and (5) ensure that, for any $w_{k i}^{\circ} \in W_{k i}$, there exist $w_{k j} \in W_{k j}, j \in N \backslash i$ such that $\sum_{j \in N \backslash i} w_{k j}+$ $w_{k i}^{\circ}=0$ (see [7]). Namely, any values in $W_{k i}, i \in$ $N$ are meaningful (there is no ineffective subarea in $\left.\boldsymbol{W}_{k}\right)$. The set of interval weight vectors $\boldsymbol{W}_{k}$ satisfying (4) and (5) is denoted by $\mathcal{W}^{\mathrm{N}}$.

Under conditions (3), (4) and (5) as well as $\epsilon<w_{i}^{\mathrm{L}} \leq w_{i}^{\mathrm{R}}, i \in N$, we calculate a suitable $\boldsymbol{W}_{k}$, where $\epsilon$ is a very small positive number. The wider each $W_{i}$ is, the easier $W_{i}, i \in N$ satisfy (3), (4) and (5). The narrower interval weights give clearer preferences in the comparison of alternatives. Then we minimize the following total widths of interval weights $W_{i}, i \in N$ :

$$
d\left(\boldsymbol{W}_{k}\right)=\sum_{i \in N}\left(w_{i}^{\mathrm{R}}-w_{i}^{\mathrm{L}}\right)
$$


Consequently, the interval weight vector $\boldsymbol{W}$ is estimated by solving the following linear programming problem:

$$
\begin{array}{r}
\underset{\boldsymbol{W}_{k}}{\operatorname{minimize}}\left\{d\left(\boldsymbol{W}_{k}\right) \mid \boldsymbol{W}_{k} \in \mathcal{W}\left(A_{k}\right) \cap \mathcal{W}^{\mathrm{N}},\right. \\
\left.\epsilon<w_{i}^{\mathrm{L}} \leq w_{i}^{\mathrm{R}}, i \in N\right\} .
\end{array}
$$

The set of optimal solutions and the optimal value to this problem is denoted by $\mathcal{W}^{\mathrm{DM}_{k}}$ and $\hat{d}_{k}$, respectively.

Once an interval weight vector $\boldsymbol{W}_{k}$ is obtained, we define a dominance relation between alternatives under the assumption that utility scores $u_{i}\left(o_{p}\right)$ of alternatives $o_{p}$ in view of each criterion are given. Sugihara et. al [5] and Guo and Tanaka [10] proposed a dominance relation based on the overall interval scores. We use the dominance relation proposed by Entani and Inuiguchi [7] because it considers all possible weights suitable for the given matrix $A_{k}$. We use two dominance relations defined by

$$
\begin{aligned}
o_{p} \succsim_{k}^{\pi} o_{q} \Leftrightarrow & \exists \boldsymbol{W}_{k} \in \mathcal{W}^{\mathrm{DM}_{k}}, \exists \boldsymbol{w} \in \boldsymbol{W}_{k}, \mathbf{e}^{\mathrm{T}} \boldsymbol{w}=1 \\
& \sum_{i \in N} w_{i}\left(u_{i}\left(o_{p}\right)-u_{i}\left(o_{q}\right)\right) \geq 0 \\
o_{p} \succsim_{k}^{\nu} o_{q} \Leftrightarrow & \exists \boldsymbol{W}_{k} \in \mathcal{W}^{\mathrm{DM}_{k}}, \forall \boldsymbol{w} \in \boldsymbol{W}_{k}, \mathbf{e}^{\mathrm{T}} \boldsymbol{w}=1 \\
& \sum_{i \in N} w_{i}\left(u_{i}\left(o_{p}\right)-u_{i}\left(o_{q}\right)\right) \geq 0
\end{aligned}
$$

where $\mathbf{e}=(1,1, \ldots, 1) \in \mathbf{R}^{n}$.

\subsection{Group Interval AHP}

\subsubsection{Application to group decision making}

We assume there are $m$ decision makers giving their own pairwise comparison matrices $A_{k}, k \in M$. We assume utility scores $u_{i}\left(o_{p}\right)$ of alternatives $o_{p}$ in view of each criterion are given. Then we apply Interval AHP only to determine the interval weights on criteria. The individual interval weight of decision maker $k$ on the $i$-th criterion is denoted by $W_{k i}=\left[w_{k i}^{\mathrm{L}}, w_{k i}^{\mathrm{R}}\right](k \in M, i \in N)$. The individual interval weight vector is denoted by $\boldsymbol{W}_{k}=$ $\left(W_{k 1}, W_{k 2}, \ldots, W_{k n}\right)^{\mathrm{T}}$. Our problem is to obtain a consensus interval weight $W_{i}=\left[w_{i}^{\mathrm{L}}, w_{i}^{\mathrm{R}}\right]$ on the $i$-th criterion among the group of decision makers. The consensus interval weight vector is denoted by $\boldsymbol{W}=\left(W_{1}, W_{2}, \ldots, W_{n}\right)^{\mathrm{T}}$.

Entani and Inuiguchi [7] have proposed three basic approaches: the perfect incorporation approach for counting out indubitably inferior alternatives and the common ground and partial incorporation approaches for finding agreeable group preference between alternatives. In this paper, we enhance those approaches by working out conceivable compromise and refinement. In this section, we briefly describe those approaches.

\subsubsection{Perfect incorporation of all individual opinions}

The first approach is a perfect incorporation of all individual opinions [7]. In this approach, a consensus interval weight vector includes all individual interval weight vectors. Namely, a consensus interval weight vector $\boldsymbol{W}$ is calculated as an optimal solution to the following linear programming problem:

$$
\begin{aligned}
\operatorname{minimize}\{d(\boldsymbol{W}) \mid & \boldsymbol{W} \supseteq \boldsymbol{W}_{k}, \boldsymbol{W} \in \mathcal{W}^{\mathrm{N}}, \\
& \left.\boldsymbol{W}_{k} \in \mathcal{W}^{\mathrm{DM}_{k}}, k \in M\right\} .
\end{aligned}
$$

It is shown that this problem is equivalent to

$$
\text { minimize }\left\{d(\boldsymbol{W}) \mid \begin{array}{rl}
\boldsymbol{W} \supseteq \boldsymbol{W}_{k}, \\
\\
\left.\boldsymbol{W}_{k} \in \mathcal{W}^{\mathrm{DM}_{k}}, k \in M\right\} .
\end{array}\right.
$$

Let $\hat{d}_{\mathrm{P}}$ be the optimal value of (11). Then the optimal solution set is defined by

$$
\begin{array}{r}
\mathcal{W}_{\mathrm{P}}=\left\{\boldsymbol{W} \mid d(\boldsymbol{W}) \leq \hat{d}_{\mathrm{P}}, \quad \boldsymbol{W} \supseteq \boldsymbol{W}_{k},\right. \\
\left.\boldsymbol{W}_{k} \in \mathcal{W}^{\mathrm{DM}_{k}}, k \in M\right\} .
\end{array}
$$

Using the perfect incorporation consensus, we define a dominance relation between alternatives by

$$
\begin{aligned}
& o_{p} \succsim_{\mathrm{P}} o_{q} \text { if and only if } \forall \boldsymbol{W} \in \mathcal{W}_{\mathrm{P}}, \forall \boldsymbol{w} \in \boldsymbol{W} \\
& \text { such that } \mathbf{e}^{\mathrm{T}} \boldsymbol{w}=1, \sum_{i \in N} w_{i}\left(u_{i}\left(o_{p}\right)-u_{i}\left(o_{q}\right)\right) \geq 0 .
\end{aligned}
$$

This dominance relation is simply called the 'perfect dominance relation' and $o_{p} \succsim_{\mathrm{P}} o_{q}$ can be verified by solving a linear programming problem [7]. For the perfect dominance relation, we have

$$
o_{p} \succsim_{\mathrm{P}} o_{q} \text { implies } \forall k \in M, o_{p} \succsim_{k}^{\nu} o_{q} .
$$

From (14), if $o_{p} \succsim_{\mathrm{P}} o_{q}$, we know that all decision makers agree to opinion that $o_{p}$ is not worse than $o_{q}$ by all means, i.e., the opinion that $o_{p}$ is not worse than $o_{q}$ is very strongly supported. However, when there is a wide range of diverse opinions, $\boldsymbol{W} \in \mathcal{W}_{\mathrm{P}}$ tends to be very wide so that almost no dominance is obtained. In such cases, this approach does not work well. On the other hand, if decision makers have similar and precise opinions, this approach works well to narrow down the candidates by erasing dominated alternatives.

\subsubsection{Taking common ground with all individual opinions}

The second approach is taking common ground with all individual opinions [7]. In this approach, a consensus interval weight vector is included in all individual interval weight vectors. This approach works only when there is a normalized interval vector $\boldsymbol{W}$ such that $\boldsymbol{W} \subseteq \bigcap_{k \in M} \boldsymbol{W}_{k}$ for some $\boldsymbol{W}_{k} \in \mathcal{W}^{\mathrm{DM}_{k}}$, $k \in M$. We note that this condition is stronger than $\bigcap_{k \in M} \boldsymbol{W}_{k} \neq \emptyset$ for some $\boldsymbol{W}_{k} \in \mathcal{W}^{\mathrm{DM}_{k}}, k \in M$. In this approach, a consensus interval weight vector $\boldsymbol{W}$ 
is calculated as an optimal solution to the following linear programming problem:

$$
\begin{aligned}
\operatorname{maximize}\{d(\boldsymbol{W}) \mid & \boldsymbol{W} \subseteq \boldsymbol{W}_{k}, \boldsymbol{W} \in \mathcal{W}^{\mathrm{N}} \\
& \left.\boldsymbol{W}_{k} \in \mathcal{W}^{\mathrm{DM}_{k}}, k \in M\right\} .
\end{aligned}
$$

Let $\hat{d}_{\mathrm{C}}$ be the optimal value of (15). Then the optimal solution set is defined by

$$
\begin{aligned}
\mathcal{W}_{\mathrm{C}}=\{ & \left\{\boldsymbol{W} \mid d(\boldsymbol{W}) \geq \hat{d}_{\mathrm{C}}, \boldsymbol{W} \subseteq \boldsymbol{W}_{k},\right. \\
& \left.\boldsymbol{W} \in \mathcal{W}^{\mathrm{N}}, \boldsymbol{W}_{k} \in \mathcal{W}^{\mathrm{DM}_{k}}, k \in M\right\} .
\end{aligned}
$$

Two dominance relations $\succsim_{\mathrm{C}}^{\mathrm{W}}$ and $\succsim_{\mathrm{C}}^{\mathrm{S}}$ between alternatives by the common ground consensus are defined by

$$
\begin{aligned}
& o_{p} \succsim \mathrm{C} o_{q} \text { if and only if } \exists \boldsymbol{W} \in \mathcal{W}_{\mathrm{C}}, \exists \boldsymbol{w} \in \boldsymbol{W} \\
& \text { such that } \mathbf{e}^{\mathrm{T}} \boldsymbol{w}=1, \sum_{i \in N} w_{i}\left(u_{i}\left(o_{p}\right)-u_{i}\left(o_{q}\right)\right) \geq 0,
\end{aligned}
$$

$o_{p} \succsim_{\mathrm{C}}^{\mathrm{S}} o_{q}$ if and only if $\forall \boldsymbol{W} \in \mathcal{W}_{\mathrm{C}}, \forall \boldsymbol{w} \in \boldsymbol{W}$ such that $\mathbf{e}^{\mathrm{T}} \boldsymbol{w}=1, \sum_{i \in N} w_{i}\left(u_{i}\left(o_{p}\right)-u_{i}\left(o_{q}\right)\right) \geq 0$.

Those relations are called a 'weak common dominance relation' and a 'strong common dominance relation', respectively. $o_{p} \succsim_{\mathrm{C}}^{\mathrm{W}} o_{q}$ and $o_{p} \succsim_{\mathrm{C}}^{\mathrm{S}} o_{q}$ can be verified by solving linear programming problems. For those dominance relations,

$$
\begin{aligned}
& o_{p} \succsim_{\mathrm{C}}^{\mathrm{S}} o_{q} \text { implies } o_{p} \succsim_{\mathrm{C}}^{\mathrm{W}} o_{q}, \\
& o_{p} \succsim_{\mathrm{C}}^{\mathrm{W}} o_{q} \text { implies } \forall k \in M, o_{p} \succsim_{k}^{\pi} o_{q}, \\
& o_{p} \succsim_{\mathrm{C}}^{\mathrm{W}} o_{q} \text { implies } o_{q} \succsim_{\mathrm{C}}^{\mathrm{S}} o_{p} .
\end{aligned}
$$

If $o_{p} \succsim \mathrm{W} o_{q}$, we know that all decision makers agree to the opinion that $o_{p}$ is not worse than $o_{q}$ in some way, i.e., the opinion that $o_{p}$ is not worse than $o_{q}$ can be supported somehow by all decision makers. If $o_{p} \succsim_{\mathrm{C}}^{\mathrm{S}} o_{q}$, we also have $o_{p} \succsim_{\mathrm{C}}^{\mathrm{W}} o_{q}$, and thus all decision makers can somehow support the opinion that $o_{p}$ is not worse than $o_{q}$. However $o_{p} \succsim_{\mathrm{C}}^{\mathrm{S}} o_{q}$ is usually stronger than $o_{p} \succsim \mathrm{C} \quad o_{q}$ because $o_{p} \succsim \mathrm{C} o_{q}$ implies $o_{p}$ is not worse than $o_{q}$ for all $\boldsymbol{w} \in \tilde{U} \mathcal{W}_{C}$ while $o_{p} \succsim \mathrm{W} o_{q}$ implies $o_{p}$ is not worse than $o_{q}$ for at least one $\boldsymbol{w} \in \bigcup \mathcal{W}_{\mathrm{C}} \cdot o_{p} \succsim_{\mathrm{C}}^{\mathrm{S}} o_{q}$ is equivalent to $o_{p} \succsim \mathrm{C} o_{q}$ when $\bigcup \mathcal{W}_{\mathrm{C}}$ is a singleton, i.e., a singleton $\{\boldsymbol{w}\}$ is a unique member of $\mathcal{W}_{\mathrm{C}}$.

We may have $o_{p} \succsim_{\mathrm{C}}^{\mathrm{W}} o_{q}$ and $o_{q} \succsim_{\mathrm{C}}^{\mathrm{W}} o_{p}$ at the same time. This makes the comparison between alternatives $o_{p}$ and $o_{q}$ difficult. Then we use strong common dominance relation $\succsim_{\mathrm{C}}^{\mathrm{S}}$ for finding agreeable group preference between alternatives.

\subsubsection{Partial incorporation of all individual opinions}

The third approach is a partial incorporation of all individual opinions [7]. In this approach, a consensus interval weight vector is a normalized interval vector intersecting with all individual interval weight vectors. Namely, a consensus interval weight vector $\boldsymbol{W}$ is calculated as an optimal solution to the following linear programming problem:

$$
\begin{array}{r}
\operatorname{minimize}\left\{d(\boldsymbol{W}) \mid \boldsymbol{w}_{k} \in \boldsymbol{W} \cap \boldsymbol{W}_{k}, \boldsymbol{W} \in \mathcal{W}^{\mathrm{N}},\right. \\
\left.\boldsymbol{W}_{k} \in \mathcal{W}^{\mathrm{DM}_{k}}, k \in M, \mathbf{e}^{\mathrm{T}} \boldsymbol{w}_{k}=1\right\} .
\end{array}
$$

It is shown that this problem is equivalent to

$$
\begin{aligned}
\operatorname{minimize}\{ & d(\boldsymbol{W}) \mid \boldsymbol{w}_{k} \in \boldsymbol{W} \cap \boldsymbol{W}_{k}, \\
& \left.\boldsymbol{W}_{k} \in \mathcal{W}^{\mathrm{DM}_{k}}, k \in M, \mathbf{e}^{\mathrm{T}} \boldsymbol{w}_{k}=1\right\} .
\end{aligned}
$$

Let $\hat{d}_{\mathrm{Q}}$ be the optimal value of (23). Then the optimal solution set is defined by

$$
\begin{aligned}
& \mathcal{W}_{\mathrm{Q}}=\left\{\boldsymbol{W} \mid d(\boldsymbol{W}) \leq \hat{d}_{\mathrm{Q}}, \boldsymbol{w}_{k} \in \boldsymbol{W} \cap \boldsymbol{W}_{k},\right. \\
& \left.\boldsymbol{W}_{k} \in \mathcal{W}^{\mathrm{DM}_{k}}, k \in M, \mathbf{e}^{\mathrm{T}} \boldsymbol{w}_{k}=1\right\} \text {. }
\end{aligned}
$$

Using the partial incorporation consensus, we define a dominance relation between alternatives by

$$
\begin{aligned}
& o_{p} \succsim_{\mathrm{Q}} o_{q} \text { if and only if } \forall \boldsymbol{W} \in \mathcal{W}_{\mathrm{Q}}, \forall \boldsymbol{w} \in \boldsymbol{W} \\
& \text { such that } \mathbf{e}^{\mathrm{T}} \boldsymbol{w}=1, \sum_{i \in N} w_{i}\left(u_{i}\left(o_{p}\right)-u_{i}\left(o_{q}\right)\right) \geq 0 .
\end{aligned}
$$

This dominance relation is called the "partial dominance relation'. $o_{p} \succsim_{\mathrm{Q}} o_{q}$ can be verified by solving a linear programming problem [7]. For the partial dominance relation, we have

$$
o_{p} \succsim_{\mathrm{Q}} o_{q} \text { implies } \forall k \in M, o_{p} \succsim_{k}^{\pi} o_{q} \text {. }
$$

If $o_{p} \succsim_{\mathrm{Q}} o_{q}$, we know that all decision makers agree to the opinion that $o_{p}$ is not worse than $o_{q}$ in some way, i.e., the opinion that $o_{p}$ is not worse than $o_{q}$ can be supported somehow by all decision makers. However, when there is a very wide range of diverse opinions, $\boldsymbol{W} \in \mathcal{W}_{\mathrm{Q}}$ tends to be wide so that dominance relation is ineffective. In such cases, this approach does not work well. However, by the partial dominance relation, we find agreeable preference between alternatives.

\section{Enhanced Group Interval AHP by Compromise and Refinement}

\subsection{Compromise in perfect incorporation of all individual opinions}

When the total width $\hat{d}_{\mathrm{P}}$ of consensus interval weight vector is big, the perfect dominance relation $\succsim_{\mathrm{P}}$ would not be very useful because $o_{p} \succsim_{\mathrm{P}} o_{q}$ does not hold for many pairs of alternatives. To enhance the usefulness of the obtained dominance relation, we consider compromise consensus interval weight vector. To decrease the total width of consensus interval weight vector, we ask the decision makers to compromise the perfect incorporation of their opinions by reducing the incorporated part of opinions. Let $r_{\mathrm{P}}$ be the reduction rate of incorporated part of opinions. The conventional perfect incorporation 
approach incorporate whole part of individual opinions, i.e., $r_{\mathrm{P}}=0$. We increase $r_{\mathrm{P}}$ from 0 to 1 so as to minimize the total width of compromised consensus interval weight vector and to obtain a sufficiently useful dominance relation.

Then the compromised consensus interval weight vector $\boldsymbol{V}$ is obtained by solving the following linear programming problem:

$$
\begin{aligned}
\operatorname{minimize}\{ & \left\{d(\boldsymbol{V}) \mid \boldsymbol{V} \supseteq \boldsymbol{V}_{k}, \boldsymbol{V}_{k} \subseteq \boldsymbol{W}_{k},\right. \\
& d\left(\boldsymbol{V}_{k}\right) \geq\left(1-r_{\mathrm{P}}\right) \hat{d}_{k}, \boldsymbol{V}_{k} \in \mathcal{W}^{\mathrm{N}}, \\
& \left.\boldsymbol{W}_{k} \in \mathcal{W}^{\mathrm{DM}_{k}}, k \in M\right\},
\end{aligned}
$$

where $\boldsymbol{V}_{k}$ is the incorporated part of individual interval weight vector and can be seen as compromised individual interval weight vector.

Let $\bar{d}_{\mathrm{P}}\left(r_{\mathrm{P}}\right)$ be the optimal value of Problem (27). Then the optimal solution set is defined by

$$
\begin{array}{r}
\mathcal{V}_{\mathrm{P}}\left(r_{\mathrm{P}}\right)=\left\{\boldsymbol{V} \mid d(\boldsymbol{V}) \leq \bar{d}_{\mathrm{P}}\left(r_{\mathrm{P}}\right), \boldsymbol{V} \supseteq \boldsymbol{V}_{k},\right. \\
\boldsymbol{V}_{k} \subseteq \boldsymbol{W}_{k}, d\left(\boldsymbol{V}_{k}\right) \geq\left(1-r_{\mathrm{P}}\right) \hat{d}_{k}, \\
\left.\boldsymbol{V}_{k} \in \mathcal{W}^{\mathrm{N}}, \quad \boldsymbol{W}_{k} \in \mathcal{W}^{\mathrm{DM}_{k}}, k \in M\right\} .
\end{array}
$$

Using the compromised perfect incorporation consensus, we define a dominance relation between alternatives by

$$
\begin{gathered}
o_{p} \succsim_{\mathrm{P}}^{r_{\mathrm{P}}} o_{q} \text { if and only if } \forall \boldsymbol{V} \in \mathcal{V}_{\mathrm{P}}\left(r_{\mathrm{P}}\right), \forall \boldsymbol{w} \in \boldsymbol{V} \\
\text { such that } \mathbf{e}^{\mathrm{T}} \boldsymbol{w}=1, \sum_{i \in N} w_{i}\left(u_{i}\left(o_{p}\right)-u_{i}\left(o_{q}\right)\right) \geq 0 .
\end{gathered}
$$

This dominance relation is simply called the ' $r_{\mathrm{P}}$-compromised perfect dominance relation' and $o_{p} \succsim_{\mathrm{P}}^{r_{\mathrm{P}}} o_{q}$ can be verified by solving a linear programming problem:

$$
\begin{aligned}
\operatorname{minimize} & \left\{\sum_{i \in N} w_{i}\left(u_{i}\left(o_{p}\right)-u_{i}\left(o_{q}\right)\right) \mid\right. \\
& \left.\boldsymbol{w} \in \boldsymbol{V}, \boldsymbol{V} \in \mathcal{V}_{\mathrm{P}}\left(r_{\mathrm{P}}\right), \mathbf{e}^{\mathrm{T}} \boldsymbol{w}=1\right\} .
\end{aligned}
$$

If the optimal value of Problem (30) is non-negative, $o_{p} \succsim_{\mathrm{P}}^{r_{\mathrm{P}}} o_{q}$ holds.

When $0<r_{\mathrm{P}}^{1}<r_{\mathrm{P}}^{2}<1$, we have

$$
\begin{aligned}
& \hat{d}_{\mathrm{P}}=\bar{d}_{\mathrm{P}}(0) \geq \bar{d}_{\mathrm{P}}\left(r_{\mathrm{P}}^{1}\right) \geq \bar{d}_{\mathrm{P}}\left(r_{\mathrm{P}}^{2}\right) \geq \hat{d}_{\mathrm{Q}}=\bar{d}_{\mathrm{P}}(1), \\
& o_{p} \succsim_{\mathrm{P}} o_{q} \text { if and only if } o_{p} \succsim_{\mathrm{P}}^{0} o_{q}, \\
& o_{p} \succsim_{\mathrm{Q}} o_{q} \text { if and only if } o_{p} \succsim_{\mathrm{P}}^{1} o_{q}, \\
& o_{p} \succsim_{\mathrm{P}} o_{q} \text { implies } o_{p} \succsim_{\mathrm{P}}^{r_{\mathrm{P}}^{1}} o_{q}, o_{p} \succsim_{\mathrm{P}}^{r_{\mathrm{P}}^{2}} o_{q} .
\end{aligned}
$$

From (34), $r_{\mathrm{P}}$-compromised perfect dominance relation refines the perfect dominance relation. However, we do not necessarily have $o_{p} \succsim_{\mathrm{P}}$ $o_{q}^{r_{\mathrm{P}}^{1}}$ implies $o_{p} \succsim_{\mathrm{P}}^{r_{\mathrm{P}}^{1}} o_{q}$. This is because the optimal solution of Problem (27) may jump from an extreme point to another by a change of $r_{\mathrm{P}}$.

\subsection{Refining the strong common dominance relation}

When there is a normalized interval vector $\boldsymbol{W}$ such that $\boldsymbol{W} \subseteq \bigcap_{k \in M} \boldsymbol{W}_{k}$ for some $\boldsymbol{W}_{k} \in \mathcal{W}^{\mathrm{DM}_{k}}$, $k \in M$, we try to find agreeable preference between alternatives by the strong common dominance relation $\succsim \mathrm{C}$. The strong common dominance relation $\succsim_{\mathrm{C}}^{\mathrm{S}}$ does not always satisfy the completeness (comparability) so that we usually have pairs of alternatives $\left(o_{p}, o_{q}\right)$ such that $o_{p} \chi_{\mathrm{C}}^{\mathrm{S}} o_{q}$ and $o_{q} \Varangle_{\mathrm{C}}^{\mathrm{S}} o_{p}$. In this case, alternatives $o_{p}$ and $o_{q}$ are indifferent by the weak common dominance relation. Namely, decision makers hesitate to declare which alternative is better for such pairs of alternatives. To suggest a probably agreeable preference between each of such alternative pairs, we propose a refinement of the strong common dominance relation $\succsim_{\mathrm{C}}^{\mathrm{S}}$.

To refine the strong common dominance relation $\succsim_{\mathrm{C}}^{\mathrm{S}}$, we suppose a probable revision of the individual interval weight vector. More concretely, we suppose that each decision maker narrows down his/her interval weight vector gradually focusing on a normalized weight vector located in his/her interval weight vector. We presume that each decision maker will select a normalized weight vector (focus point) near the center of his/her interval weight vector. Under this presumption, we solve the following linear programming problem to define the focus point:

$$
\begin{gathered}
\underset{\operatorname{minimize}}{ }\left\{\sum_{k \in M} \sum_{i \in N} s_{k i} \mid d(\boldsymbol{W}) \geq \hat{d}_{\mathrm{C}},\right. \\
\boldsymbol{W} \subseteq \boldsymbol{W}_{k}, \boldsymbol{W} \in \mathcal{W}^{\mathrm{N}}, \boldsymbol{W}_{k} \in \mathcal{W}^{\mathrm{DM}_{k}}, \\
\frac{1}{2}\left(w_{k i}^{\mathrm{L}}+w_{k i}^{\mathrm{R}}\right)-w_{k i} \leq s_{k i}, \\
w_{k i}-\frac{1}{2}\left(w_{k i}^{\mathrm{L}}+w_{k i}^{\mathrm{R}}\right) \leq s_{k i}, i \in N, \\
\left.\boldsymbol{w}_{k} \in \boldsymbol{W}_{k}, \mathbf{e}^{\mathrm{T}} \boldsymbol{w}_{k}=1, k \in M\right\},
\end{gathered}
$$

Let $\hat{s}$ be the optimal value. Considering the possibility that Problem (35) has multiple optimal solutions, the reduced consensus interval weight vector $\boldsymbol{V}$ with reduction rate $r_{\mathrm{C}}$ is calculated as an optimal solution to the following linear programming problem:

$$
\begin{aligned}
& \operatorname{maximize}\left\{d(\boldsymbol{V}) \mid \boldsymbol{V} \subseteq \boldsymbol{V}_{k}, \boldsymbol{V} \in \mathcal{V}^{\mathrm{N}},\right. \\
& d\left(\boldsymbol{V}_{k}\right) \leq\left(1-r_{\mathrm{C}}\right) \hat{d}_{k}, \boldsymbol{V}_{k} \subseteq \boldsymbol{W}_{k}, \\
& \boldsymbol{W} \subseteq \boldsymbol{W}_{k}, \boldsymbol{W} \in \mathcal{W}^{\mathrm{N}}, \boldsymbol{W}_{k} \in \mathcal{W}^{\mathrm{DM}_{k}}, \\
& \sum_{k \in M} \sum_{i \in N} s_{k i} \leq \hat{s}, d(\boldsymbol{W}) \geq \hat{d}_{\mathrm{C}}, \\
& w_{k i}-s_{k i} \leq \frac{1}{2}\left(w_{k i}^{\mathrm{L}}+w_{k i}^{\mathrm{R}}\right) \leq w_{k i}+s_{k i}, \\
& w_{k i}-s_{k i} \leq \frac{1}{2}\left(v_{k i}^{\mathrm{L}}+v_{k i}^{\mathrm{R}}\right) \leq w_{k i}+s_{k i}, \\
& \left.\boldsymbol{w}_{k} \in \boldsymbol{W}_{k}, \mathbf{e}^{\mathrm{T}} \boldsymbol{w}_{k}=1, i \in N, k \in M\right\} .
\end{aligned}
$$

Let $\bar{d}_{\mathrm{C}}\left(r_{\mathrm{C}}\right)$ be the optimal value of Problem (36). Then the optimal solution set to Problem (36) is 
defined by

$$
\begin{aligned}
& \mathcal{V}_{\mathrm{C}}\left(r_{\mathrm{C}}\right)=\left\{d(\boldsymbol{V}) \geq \bar{d}_{\mathrm{C}}\left(r_{\mathrm{C}}\right), \quad \boldsymbol{V} \subseteq \boldsymbol{V}_{k},\right. \\
& \boldsymbol{V} \in \mathcal{V}^{\mathrm{N}}, d\left(\boldsymbol{V}_{k}\right) \leq\left(1-r_{\mathrm{C}}\right) \hat{d}_{k}, \boldsymbol{W} \subseteq \boldsymbol{W}_{k}, \\
& \boldsymbol{V}_{k} \subseteq \boldsymbol{W}_{k}, \boldsymbol{W} \in \mathcal{W}^{\mathrm{N}}, \boldsymbol{W}_{k} \in \mathcal{W}^{\mathrm{DM}}, \\
& \sum_{k \in M} \sum_{i \in N} s_{k i} \leq \hat{s}, d(\boldsymbol{W}) \geq \hat{d}_{\mathrm{C}}, \\
& w_{k i}-s_{k i} \leq \frac{1}{2}\left(w_{k i}^{\mathrm{L}}+w_{k i}^{\mathrm{R}}\right) \leq w_{k i}+s_{k i}, \\
& w_{k i}-s_{k i} \leq \frac{1}{2}\left(v_{k i}^{\mathrm{L}}+v_{k i}^{\mathrm{R}}\right) \leq w_{k i}+s_{k i}, \\
& \left.\boldsymbol{w}_{k} \in \boldsymbol{W}_{k}, \mathbf{e}^{\mathrm{T}} \boldsymbol{w}_{k}=1, i \in N, k \in M\right\} .
\end{aligned}
$$

$\boldsymbol{V} \in \mathcal{V}_{\mathrm{C}}\left(r_{\mathrm{C}}\right)$ is the reduced consensus interval weight vector which is composed of a common part among the refined individual interval weight vectors $\boldsymbol{V}_{k}, k \in M$. Each decision maker is assumed to refine the individual interval weight vector into an interval weight vector with $100 r_{\mathrm{C}} \%$ narrower width whose center value vector is similar to the original center. In other words, the larger $r_{\mathrm{C}}$, the more robust against the individual refinement a normalized weight vector $\boldsymbol{v} \in \boldsymbol{V} \in \mathcal{V}_{\mathrm{C}}\left(r_{\mathrm{C}}\right)$.

Using the refined common ground consensus, we define a dominance relation between alternatives by

$o_{p} \succsim_{\mathrm{C}}^{r_{\mathrm{C}}} o_{q}$ if and only if $\forall \boldsymbol{V} \in \mathcal{V}_{\mathrm{C}}\left(r_{\mathrm{C}}\right), \forall \boldsymbol{w} \in \boldsymbol{V}$

$$
\text { such that } \mathbf{e}^{\mathrm{T}} \boldsymbol{w}=1, \sum_{i \in N} w_{i}\left(u_{i}\left(o_{p}\right)-u_{i}\left(o_{q}\right)\right) \geq 0 \text {. }
$$

This dominance relation is simply called the ' $r_{\mathrm{C}^{-}}$ refined strong common dominance relation' and $o_{p} \succsim_{\mathrm{C}}^{r_{\mathrm{C}}} o_{q}$ can be verified by solving a linear programming problem:

$$
\begin{aligned}
\operatorname{minimize} & \left\{\sum_{i \in N} w_{i}\left(u_{i}\left(o_{p}\right)-u_{i}\left(o_{q}\right)\right) \mid\right. \\
& \left.\boldsymbol{w} \in \boldsymbol{V}, \boldsymbol{V} \in \mathcal{V}_{\mathrm{C}}\left(r_{\mathrm{C}}\right), \mathbf{e}^{\mathrm{T}} \boldsymbol{w}=1\right\} .
\end{aligned}
$$

If the optimal value of Problem (39) is non-negative, $o_{p} \succsim_{\mathrm{C}}^{r_{\mathrm{C}}} o_{q}$ holds.

When $0<r_{\mathrm{C}}^{1}<r_{\mathrm{C}}^{2}<1$, we have

$$
\begin{aligned}
& \hat{d}_{\mathrm{C}}=\bar{d}_{\mathrm{C}}(0) \geq \bar{d}_{\mathrm{C}}\left(r_{\mathrm{C}}^{1}\right) \geq \bar{d}_{\mathrm{P}}\left(r_{\mathrm{C}}^{2}\right), \\
& o_{p} \succsim_{\mathrm{C}} o_{q} \text { if and only if } o_{p} \succsim_{\mathrm{C}}^{0} o_{q}, \\
& o_{p} \succsim_{\mathrm{C}} o_{q} \text { implies } o_{p} \succsim_{\mathrm{C}}^{r_{\mathrm{C}}^{1}} o_{q}, o_{p} \succsim_{\mathrm{C}}^{r_{\mathrm{C}}^{2}} o_{q} .
\end{aligned}
$$

From (42), we know that $r_{\mathrm{C}}$-refined strong common dominance relation refines the strong common dominance relation as its name suggests. However, we do not always have $o_{p} \succsim_{\mathrm{C}}^{r_{\mathrm{C}}^{1}} o_{q}$ implies $o_{p} \succsim_{\mathrm{C}}^{r_{\mathrm{C}}^{2}} o_{q}$.

\subsection{Compromise in partial incorporation of all individual opinions}

When the total width $\hat{d}_{\mathrm{Q}}$ of consensus interval weight vector is not sufficiently small, the partial dominance relation $\succsim_{\mathrm{Q}}$ would not find any strict domination for several pairs of alternatives. Especially when decision makers' opinions widely spread, many pairs of alternatives are incomparable. In order to obtain a clearer ranking, we refine the partial dominance. To decrease the total width of consensus interval weight vector, we ask the decision makers to compromise on the individual interval weight vectors by relaxing the minimal total widths. Let $r_{\mathrm{Q}}$ be the relaxation rate of the individual total width. By this relaxation, more interval weight vectors become candidates for the individual interval weight vector and we assume that the decision maker can accept each of them.

Then the compromised consensus interval weight vector $\boldsymbol{V}$ is obtained by solving the following linear programming problem:

$$
\begin{aligned}
\operatorname{minimize} & \left\{d(\boldsymbol{V}) \mid \boldsymbol{w}_{k} \in \boldsymbol{V} \cap \boldsymbol{V}_{k},\right. \\
\mathbf{e}^{\mathrm{T}} \boldsymbol{w}_{k}=1, d\left(\boldsymbol{V}_{k}\right) \leq\left(1+r_{\mathrm{Q}}\right) \hat{d}_{k}, & \left.\boldsymbol{V}_{k} \in \mathcal{W}\left(A_{k}\right) \cap \mathcal{W}^{\mathrm{N}}, k \in M\right\},
\end{aligned}
$$

where $\boldsymbol{V}_{k}$ is the compromised individual interval weight vector. We note that $\boldsymbol{V} \in \mathcal{W}^{\mathrm{N}}$ is satisfied at the optimal solution. When $r_{\mathrm{Q}}=0, \boldsymbol{V}$ and $\boldsymbol{V}_{k}$, $k \in M$ equal to the usual common ground consensus $\boldsymbol{W}$ and usual individual interval weight vectors $\boldsymbol{W}_{k}$, $k \in M$, respectively. We consider $r_{\mathrm{Q}}>0$. When $r_{\mathrm{Q}}$ is sufficiently large, we have $d(\boldsymbol{V})=0$ at the optimal solution to Problem (43).

Let $\bar{d}_{\mathrm{Q}}\left(r_{\mathrm{Q}}\right)$ be the optimal value of Problem (43). Then the optimal solution set is defined by

$$
\begin{aligned}
& \mathcal{V}_{\mathrm{Q}}\left(r_{\mathrm{Q}}\right)=\left\{\boldsymbol{V} \mid \exists \boldsymbol{w}_{k} \in \boldsymbol{V} \cap \boldsymbol{V}_{k},\right. \\
& d(\boldsymbol{V}) \leq \bar{d}_{\mathrm{Q}}\left(r_{\mathrm{Q}}\right), d\left(\boldsymbol{V}_{k}\right) \leq\left(1+r_{\mathrm{Q}}\right) \hat{d}_{k}, \\
& \left.\mathbf{e}^{\mathrm{T}} \boldsymbol{w}_{k}=1, \boldsymbol{V}_{k} \in \mathcal{W}\left(A_{k}\right) \cap \mathcal{W}^{\mathrm{N}}, k \in M\right\} .
\end{aligned}
$$

Using the compromised partial incorporation consensus, we define a dominance relation between alternatives by

$$
\begin{aligned}
& o_{p} \succsim_{\mathrm{Q}}^{r_{\mathrm{Q}}} o_{q} \text { if and only if } \forall \boldsymbol{V} \in \mathcal{V}_{\mathrm{Q}}\left(r_{\mathrm{Q}}\right), \forall \boldsymbol{w} \in \boldsymbol{V} \\
& \text { such that } \mathbf{e}^{\mathrm{T}} \boldsymbol{w}=1, \sum_{i \in N} w_{i}\left(u_{i}\left(o_{p}\right)-u_{i}\left(o_{q}\right)\right) \geq 0 .
\end{aligned}
$$

This dominance relation is simply called the ' $r_{\mathrm{Q}}$-compromised partial dominance relation' and $o_{p} \succsim_{\mathrm{Q}}^{r_{\mathrm{Q}}} o_{q}$ can be verified by solving a linear programming problem:

$$
\begin{aligned}
\operatorname{minimize} & \left\{\sum_{i \in N} w_{i}\left(u_{i}\left(o_{p}\right)-u_{i}\left(o_{q}\right)\right) \mid\right. \\
& \left.\boldsymbol{w} \in \boldsymbol{V}, \boldsymbol{V} \in \mathcal{V}_{\mathrm{Q}}\left(r_{\mathrm{Q}}\right), \mathbf{e}^{\mathrm{T}} \boldsymbol{w}=1\right\} .
\end{aligned}
$$

If the optimal value of Problem (46) is non-negative, $o_{p} \succsim_{\mathrm{Q}}^{r_{\mathrm{Q}}} o_{q}$ holds.

When $0<r_{\mathrm{Q}}^{1}<r_{\mathrm{Q}}^{2}$, we have

$$
\begin{aligned}
& \hat{d}_{\mathrm{Q}}=\bar{d}_{\mathrm{Q}}(0) \geq \bar{d}_{\mathrm{Q}}\left(r_{\mathrm{Q}}^{1}\right) \geq \bar{d}_{\mathrm{Q}}\left(r_{\mathrm{Q}}^{2}\right), \\
& o_{p} \succsim_{\mathrm{Q}} o_{q} \text { if and only if } o_{p} \succsim_{\mathrm{Q}}^{0} o_{q}, \\
& o_{p} \succsim_{\mathrm{Q}} o_{q} \text { implies } o_{p} \succsim_{\mathrm{Q}}^{r_{\mathrm{Q}}^{1}} o_{q}, o_{p} \succsim_{\mathrm{Q}}^{r_{\mathrm{Q}}^{2}} o_{q} .
\end{aligned}
$$

From (49), $r_{\mathrm{Q}}$-compromised partial dominance relation refines the partial dominance relation. 


\section{Numerical Examples}

In this section, we demonstrate how the proposed approach refines the dominance relations. We consider the comparison of three alternatives $o_{1}, o_{2}$ and $o_{3}$ by four criteria $C_{1}, C_{2}, C_{3}$ and $C_{4}$ by a group of three decision makers $D M_{1}, D M_{2}$ and $D M_{3}$. The utility scores of alternatives are given as in Table 1 .

\subsection{Case where no common ground exists}

We consider pairwise comparison matrices of three decision makers given as in Table 2. The minimal total width $\hat{d}_{k}$ is shown at the bottom of each decision maker's pairwise comparison matrix in Table 2 .

Applying the perfect incorporation approach with a suitable compromise, we obtain $r_{\mathrm{P}}$-compromised perfect dominance relations from optimal values of Problems (30) for all possible ordered pairs of alternatives. Varying $r_{\mathrm{P}} \in[0,1]$ with step 0.2 , we calculated the optimal values of Problems (30) for all possible ordered pairs of alternatives. In Table 3 , the optimal values of Problems (30) for all possible ordered pairs of alternatives only for $r_{\mathrm{P}}=0,0.6$ and 1 are shown. On the left upper corner of each small table of Table 3 , the value of $r_{\mathrm{P}}$ is shown. As shown in Table 3 , all values are negative. This implies that opinion differences among three decision makers are rather large, so that we did not succeed in refining the perfect dominance relation.

Because common ground does not exist in this example, we apply the partial incorporation approach with a suitable compromise. Choosing several values of $r_{\mathrm{Q}} \in[0,0.17]$, we calculated the optimal values of Problems (46) for all possible ordered pairs of alternatives. In Table 4, the optimal values of Problem (46) only for $r_{\mathrm{Q}}=0,0.1$ and 0.16 are shown. As shown in Table 4, the partial dominance relation is refined gradually. Moreover we recognize that $o_{3}$ is better than the others from the compromised partial incorporation consensus with $r_{\mathrm{Q}}=0.16$. However, in this example, a total order is not obtained because we have multiple optimal solutions to Problem (43) when $r_{\mathrm{Q}}=0.17$ and the optimal values of Problem (46) for comparison of $o_{1}$ and $o_{2}$ with $r_{\mathrm{Q}}=0.17$ are both negative.

\subsection{Case where common ground exists}

We consider pairwise comparison matrices of three decision makers given as in Table 5. The minimal total width $\hat{d}_{k}$ is shown at the bottom of each decision maker's pairwise comparison matrix in Table 5 . From values of $\hat{d}_{k}, k=1,2,3$, we know that those decision makers' opinions are vague.

Applying the perfect incorporation approach with a suitable compromise, we obtain $r_{\mathrm{P}}$-compromised perfect dominance relations from optimal values of Problems (30) for all possible ordered pairs of alternatives. Varying $r_{\mathrm{P}} \in[0,1]$ with step 0.2 , we calculated the optimal values of Problems (30) for
Table 1: Utility scores

\begin{tabular}{c|cccc}
\hline \multirow{2}{*}{} & \multicolumn{4}{|c}{ Criterion } \\
\cline { 2 - 5 } & $C_{1}$ & $C_{2}$ & $C_{3}$ & $C_{4}$ \\
\hline$o_{1}$ & 0.7 & 1 & 0.3 & 0.2 \\
$o_{2}$ & 0.75 & 0.1 & 1 & 1 \\
$o_{3}$ & 1 & 0.3 & 0.45 & 0.8 \\
\hline
\end{tabular}

Table 2: Individual pairwise comparison matrices (1)

\begin{tabular}{|c|c|c|c|c|}
\hline$D M_{1}$ & $\overline{C_{1}}$ & $C_{2}$ & $C_{3}$ & $C_{4}$ \\
\hline$C_{1}$ & 1 & 2 & 3 & 4 \\
\hline$C_{2}$ & - & 1 & 2 & 3 \\
\hline$C_{3}$ & - & - & 1 & 2 \\
\hline$C_{4}$ & - & - & - & 1 \\
\hline & \multicolumn{4}{|c|}{$\hat{d}_{1}=0.0833$} \\
\hline$D M_{2}$ & $C_{1}$ & $C_{2}$ & $C_{3}$ & $C_{4}$ \\
\hline$C_{1}$ & 1 & 3 & 3 & 4 \\
\hline$C_{2}$ & - & 1 & 3 & 3 \\
\hline$C_{3}$ & - & - & 1 & 4 \\
\hline \multirow[t]{2}{*}{$C_{4}$} & - & - & - & 1 \\
\hline & \multicolumn{4}{|c|}{$\hat{d}_{2}=0.2381$} \\
\hline$D M_{3}$ & $C_{1}$ & $C_{2}$ & $C_{3}$ & $C_{4}$ \\
\hline$C_{1}$ & 1 & 1 & 4 & 6 \\
\hline$C_{2}$ & - & 1 & 1 & 2 \\
\hline$C_{3}$ & - & - & 1 & 3 \\
\hline \multirow[t]{2}{*}{$C_{4}$} & - & - & - & 1 \\
\hline & \multicolumn{4}{|c|}{$\hat{d}_{3}=0.3496$} \\
\hline
\end{tabular}

all possible ordered pairs of alternatives. In Table 6 , the optimal values of Problems (30) for all possible ordered pairs of alternatives only for $r_{\mathrm{P}}=0,0.6$ and 1 are shown. On the left upper corner of each small table of Table 3 , the value of $r_{\mathrm{P}}$ is shown. As shown in Table 6 , most of values are negative but the value at $\left(o_{1}, o_{2}\right)$ of table for $r_{\mathrm{P}}=1$ is positive. This fact implies that the perfect dominance relation is refined by the compromise. However, for the other pairs, the individual opinions are still different or vague but we cannot make further compromise in the perfect incorporation approach.

In this example, a common ground exists. Then we apply the common ground approach with a suitable refinement of the dominance relation. Varying $r_{\mathrm{C}} \in[0,0.6]$ with step 0.2 , we calculated the optimal values of Problems (39) for all possible ordered pairs of alternatives. In Table 7 , the optimal values of Problems (39) for all possible ordered pairs of alternatives only for $r_{\mathrm{C}}=0,0.4$ and 0.6 are shown. On the left upper corner of each small table of Table 7 , the value of $r_{\mathrm{C}}$ is shown. As shown in Table 7 , the number of positive components in a small table increases gradually as $r_{\mathrm{C}}$ increases. This implies that the strong common dominance relation is gradually refined and finally it becomes a total order at $r_{\mathrm{C}}=0.6$. If the decision maker accepts this refinement, we suppose that the opinion telling $o_{1}$ is the best and $o_{2}$ is the worst is agreeable among 
Table 3: Perfect dominance (1)

\begin{tabular}{c|ccc}
\hline 0 & $o_{1}$ & $o_{2}$ & $o_{3}$ \\
\hline$o_{1}$ & - & -0.1347 & -0.1381 \\
$o_{2}$ & -0.2386 & - & -0.1560 \\
$o_{3}$ & -0.1017 & -0.0206 & - \\
\hline \hline 0.6 & $o_{1}$ & $O_{2}$ & $o_{3}$ \\
\hline$o_{1}$ & - & -0.1190 & -0.1270 \\
$o_{2}$ & -0.1547 & - & -0.1032 \\
$o_{3}$ & -0.0688 & -0.0122 & - \\
\hline \hline 1 & $O_{1}$ & $O_{2}$ & $O_{3}$ \\
\hline$o_{1}$ & - & -0.1151 & -0.1287 \\
$o_{2}$ & -0.1547 & - & -0.1032 \\
$o_{3}$ & -0.0688 & -0.0122 & - \\
\hline \multicolumn{4}{|c}{}
\end{tabular}

Table 4: Partial dominance

\begin{tabular}{c|ccc}
\hline 0 & $o_{1}$ & $o_{2}$ & $o_{3}$ \\
\hline$o_{1}$ & - & -0.1151 & -0.1287 \\
$o_{2}$ & -0.1477 & - & -0.1105 \\
$o_{3}$ & -0.0705 & -0.0098 & - \\
\hline \hline 0.1 & $o_{1}$ & $o_{2}$ & $o_{3}$ \\
\hline$o_{1}$ & - & -0.0479 & -0.0957 \\
$o_{2}$ & -0.0828 & - & -0.0993 \\
$o_{3}$ & -0.0007 & 0.0347 & - \\
\hline \hline 0.16 & $o_{1}$ & $o_{2}$ & $O_{3}$ \\
\hline$o_{1}$ & - & -0.0132 & -0.0737 \\
$o_{2}$ & -0.0390 & - & -0.0812 \\
$o_{3}$ & 0.0338 & 0.0577 & - \\
\hline
\end{tabular}

Table 5: Individual pairwise comparison matrices $(2)$

\begin{tabular}{c|cccc}
\hline$D M_{1}$ & $C_{1}$ & $C_{2}$ & $C_{3}$ & $C_{4}$ \\
\hline$C_{1}$ & 1 & 3 & 1 & 5 \\
$C_{2}$ & - & 1 & 3 & 7 \\
$C_{3}$ & - & - & 1 & 9 \\
$C_{4}$ & - & - & - & 1 \\
\hline & \multicolumn{2}{|c}{$\hat{d}_{1}=0.5614$} & \\
\hline \hline$D M_{2}$ & $C_{1}$ & $C_{2}$ & $C_{3}$ & $C_{4}$ \\
\hline$C_{1}$ & 1 & 1 & 5 & 3 \\
$C_{2}$ & - & 1 & 1 & 9 \\
$C_{3}$ & - & - & 1 & 7 \\
$C_{4}$ & - & - & - & 1 \\
\hline & $\hat{d}_{2}=0.4070$ & \\
\hline \hline$D M_{3}$ & $C_{1}$ & $C_{2}$ & $C_{3}$ & $C_{4}$ \\
\hline$C_{1}$ & 1 & 3 & 1 & 3 \\
$C_{2}$ & - & 1 & 5 & 9 \\
$C_{3}$ & - & - & 1 & 7 \\
$C_{4}$ & - & - & - & 1 \\
\hline & \multicolumn{5}{|c}{$\hat{d}_{3}=0.6576$} & \\
\hline
\end{tabular}

Table 6: Perfect dominance (2)

\begin{tabular}{c|ccc}
\hline 0 & $o_{1}$ & $o_{2}$ & $o_{3}$ \\
\hline$o_{1}$ & - & -0.2803 & -0.1671 \\
$o_{2}$ & -0.2296 & - & -0.1413 \\
$o_{3}$ & -0.1246 & -0.1344 & - \\
\hline \hline 0.6 & $o_{1}$ & $o_{2}$ & $O_{3}$ \\
\hline$o_{1}$ & - & -0.0961 & -0.0640 \\
$o_{2}$ & -0.1632 & - & -0.0857 \\
$o_{3}$ & -0.0896 & -0.0334 & - \\
\hline \hline 1 & $o_{1}$ & $O_{2}$ & $O_{3}$ \\
\hline$o_{1}$ & - & 0.0006 & -0.0046 \\
$o_{2}$ & -0.1391 & - & -0.0686 \\
$o_{3}$ & -0.0829 & -0.0070 & - \\
\hline
\end{tabular}

Table 7: Strong common dominance

\begin{tabular}{c|ccc}
\hline 0 & $o_{1}$ & $o_{2}$ & $o_{3}$ \\
\hline$o_{1}$ & - & 0.0006 & -0.0046 \\
$o_{2}$ & -0.1391 & - & -0.0686 \\
$o_{3}$ & -0.0829 & -0.0070 & - \\
\hline \hline 0.4 & $o_{1}$ & $o_{2}$ & $o_{3}$ \\
\hline$o_{1}$ & - & 0.0040 & -0.0029 \\
$o_{2}$ & -0.1045 & - & -0.0539 \\
$o_{3}$ & -0.0703 & 0.0068 & - \\
\hline \hline 0.6 & $o_{1}$ & $o_{2}$ & $o_{3}$ \\
\hline$o_{1}$ & - & 0.0365 & 0.0144 \\
$o_{2}$ & -0.0596 & - & -0.0329 \\
$o_{3}$ & -0.0464 & 0.0221 & - \\
\hline
\end{tabular}

the group of decision makers. This is a case where the proposed refinement works very well.

\section{Concluding Remarks}

We have extended three approaches of Group Interval AHP by introducing compromise and refinement. Depending on the approach, methods for compromise and refinement are different. By compromise, we show that the consensus dominance relation is refined. The consensus dominance relations are obtained by solving linear programming problems. The properties of the refined dominance relations are shown. Giving the reasonable suggestion to the decision makers toward the preference revision in view of all opinions is one of the future topic related to this study.

\section{Acknowledgment}

This work was supported by JSPS KAKENHI Grant Number 26350423.

\section{References}

[1] T. L. Saaty, The Analytic Hierarchy Process, McGraw-Hill, New York, 1980.

[2] P. J. M. van Laarhoven and W. Pedrycz, A fuzzy extension of Saaty's priority theory, Fuzzy Sets and Systems, 11:199-227, Elsevier, 1983.
[3] J. J. Buckley, Fuzzy hierarchical analysis, Fuzzy Sets and Systems, 17:233-247, Elsevier, 1985.

[4] A. Arbel, Approximate articulation of preference and priority derivation, European Journal of Operational Research, 43:317-326, Elsevier, 1989.

[5] K. Sugihara and H. Tanaka, Interval evaluations in the analytic hierarchy process by possibilistic analysis, Computational Intelligence, 17:567579, Wiley, 2001.

[6] K. Sugihara, H. Ishii, and H. Tanaka, Interval priorities in AHP by interval regression analysis, European Journal of Operational Research, 158:745-754, Elsevier, 2004.

[7] T. Entani and M. Inuiguchi, Pairwise comparison based interval analysis for group decision aiding with multiple criteria, Fuzzy Sets and Systems, Elsevier, 2015 (in press).

[8] E. Herrera-Viedma, F. J. Cabrerizo, J. Kacprzyk, and W. Pedrycz, A review of soft consensus models in a fuzzy environment, Information Fusion, 17:4-13, Elsevier, 2014.

[9] H. Tanaka, K. Nagasaka, and I. Hayashi, Interval regression analysis by possibilistic measures (in Japanese), The Japanese Journal of Behaviormetrics, 16:1-7, BSJ, 1988.

[10] P. Guo and H. Tanaka, Decision making with interval probabilities, European Journal of $\mathrm{Op}$ erational Research, 203:444-454, Elsevier, 2010. 